\title{
Long-Term Effects of Maternal Deprivation on Cholinergic System in Rat Brain
}

\author{
Branka Marković, ${ }^{1}$ Nevena V. Radonjić, ${ }^{2}$ Milan Aksić, ${ }^{3}$ \\ Branislav Filipović, ${ }^{3}$ and Nataša Petronijević ${ }^{2}$ \\ ${ }^{1}$ Faculty of Sport and Physical Education, University of Belgrade, Blagoja Parovića 156, 11000 Belgrade, Serbia \\ ${ }^{2}$ Institute of Clinical and Medical Biochemistry, Faculty of Medicine, University of Belgrade, Pasterova 2, 11000 Belgrade, Serbia \\ ${ }^{3}$ Institute of Anatomy "Niko Miljanić", Faculty of Medicine, University of Belgrade, Dr. Subotića 4, 11000 Belgrade, Serbia \\ Correspondence should be addressed to Nataša Petronijević; natasapetronijevic@yahoo.com
}

Received 8 December 2013; Revised 27 January 2014; Accepted 28 January 2014; Published 10 March 2014

Academic Editor: Igor Jakovcevski

Copyright (C) 2014 Branka Marković et al. This is an open access article distributed under the Creative Commons Attribution License, which permits unrestricted use, distribution, and reproduction in any medium, provided the original work is properly cited.

\begin{abstract}
Numerous clinical studies have demonstrated an association between early stressful life events and adult life psychiatric disorders including schizophrenia. In rodents, early life exposure to stressors such as maternal deprivation (MD) produces numerous hormonal, neurochemical, and behavioral changes and is accepted as one of the animal models of schizophrenia. The stress induces acetylcholine (Ach) release in the forebrain and the alterations in cholinergic neurotransmitter system are reported in schizophrenia. The aim of this study was to examine long-term effects of maternal separation on acetylcholinesterase (AChE) activity in different brain structures and the density of cholinergic fibers in hippocampus and retrosplenial (RS) cortex. Wistar rats were separated from their mothers on the postnatal day (P) 9 for $24 \mathrm{~h}$ and sacrificed on P60. Control group of rats was bred under the same conditions, but without MD. Brain regions were collected for AChE activity measurements and morphometric analysis. Obtained results showed significant decrease of the $\mathrm{AChE}$ activity in cortex and increase in the hippocampus of MD rats. Density of cholinergic fibers was significantly increased in CA1 region of hippocampus and decreased in RS cortex. Our results indicate that MD causes long-term structure specific changes in the cholinergic system.
\end{abstract}

\section{Introduction}

Animal model of maternal deprivation (MD) is based on exposure to stress in early postnatal life. It has repeatedly been shown that early perinatal stress can cause various shortand long-term disturbances in cognitive, emotional, and other behavioral performances $[1,2]$. Nonetheless, there is evidence that early stressful life events can increase the risk of developing schizophrenia [3-5]. Schizophrenia is a chronic, severe, and disabling brain disorder. Typical symptoms of schizophrenia can be divided into positive, negative, and cognitive ones. Typical antipsychotic drugs are effective in reducing the positive symptoms, but there is no efficacy against the negative symptoms and cognitive disorder [68]. Cholinergic system is a target for drug development aimed at improving treatments $[9,10]$. Cholinergic disturbance in basal forebrain structures and their projections in schizophrenia could be notable for cognitive dysfunction given their known functional roles in conscious awareness and components of information processing, including attention, working memory, encoding memory consolidation, and retrieval $[11,12]$. Recent studies show that selective muscarinic receptor agonist (xanomeline) can improve cognitive dysfunction in patients affected with schizophrenia [13].

The stress response includes acetylcholine (Ach) release in the forebrain, which plays an important role in many cognitive functions like learning [14, 15], attention [16], memory [17], and cortical modulation of sensory information [18]. This release of Ach is responsible for physiological and emotional responses, in particular through its action on the hypothalamic-pituitary system [19], one of the main physiological systems mediating the neuroendocrine response to stress [20]. Alterations in acetylcholine neurotransmission have been commonly reported in schizophrenia [21, 22]. 
The aim of this study was to examine long-term effects of maternal separation on cholinergic system by measuring AChE activity in different brain structures and density of cholinergic fibers in the hippocampus and retrosplenial (RS) cortex of rats.

\section{Methods}

2.1. Animals and Procedures. Male and nulliparous female Wistar rats at the age of 3 months were put together in standard Plexiglas cages with sawdust $(26 \times 42 \times 15 \mathrm{~cm})$, in a temperature controlled room $\left(23 \pm 1^{\circ} \mathrm{C}\right)$. The rats were on a standard $12 \mathrm{~h}$ light/dark cycle with lights on from 7:00 to 19:00 h, with water and food available ad libitum. Two weeks later, the males were removed and the dams were checked twice daily for delivery. The day of delivery was denoted as postnatal day (P) 0. On P 9, pups from four litters were subjected to the maternal deprivation procedure according to the previously published protocol [23]. Briefly, the mothers were removed from the cage around 10:00 a.m. and placed in a single cage housed in the same room as the pups. The pups were weighed and left in the home cage at room temperature. In the control four litters, mothers were removed at P 9, pups were weighed, and mothers were returned immediately (within 3 minutes). At P 10 (24 hours later), the deprived pups were weighed again and the mothers were placed back in the home cage. In the control litters, the mothers were again removed, the pups were weighed, and the mothers were placed back in the cage. All litters were later left undisturbed except for the routine cleaning of the cages, until P 21. On P 21 , the litters were weaned. Rats were sacrificed at 2 months of age (P60). All experiments were performed on male animals.

All efforts were made to minimize animal suffering and reduce the number of animals used in the study. All experiments were carried out according to the NIH Guide for Care and Use of Laboratory Animals and were approved by the Local Bioethics Committee.

2.2. Brain Preparation for Measurements of Acetylcholinesterase Activity. Eight animals from control and eight from experimental groups were used for biochemical analysis of acetylcholinesterase activity. Four brain regions, dorsolateral frontal cortex, hippocampus, thalamus, and caudate nuclei, were dissected and the crude synaptosomal fraction was prepared according to the method of Whittaker and Barker [24-26]. Briefly, isolation of specific brain structures from individual animals was performed quickly on ice. Isolated tissue was homogenized in ice-cold buffer, $\mathrm{pH}$ 7.0, containing 0.25 M sucrose, 0.1 mM EDTA, and $50 \mathrm{mM} \mathrm{K-Na} \mathrm{phosphate}$ buffer. Homogenates were centrifuged twice at $1000 \times \mathrm{g}$ for $15 \mathrm{~min}$ at $4^{\circ} \mathrm{C}$. The supernatant was further centrifuged at $20,000 \times \mathrm{g}$ for $20 \mathrm{~min}$. Supernatant obtained by this procedure represents crude synaptosomal fraction containing membrane vesicles (microsomes) from smooth and rough endoplasmic reticulum, Golgi and plasma membrane, and all of the soluble components of the cytoplasm.
2.3. Immunohistochemistry. For morphological analysis, five male animals from the control and five from experimental groups (P 60) were anaesthetized with chloral hydrate $(3 \mathrm{mg} / \mathrm{kg}$, i. p.) and transcardially perfused with fixative (4\% formaldehyde in $0.1 \mathrm{M}$ phosphate buffer solution). The brains were postfixed for $24 \mathrm{~h}$ at $+4^{\circ} \mathrm{C}$ and cryoprotected by infiltration with sucrose for 2 days at $4^{\circ} \mathrm{C}(20 \%$ sucrose in $0.1 \mathrm{M}$ phosphate buffer). Brains were frozen by immersion in 2-methyl-butane (Fluka) precooled to $-80^{\circ} \mathrm{C}$ and stored at $-80^{\circ} \mathrm{C}$ until cutting. Serial transverse sections $(25-\mu \mathrm{m}-$ thick) were cut on a cryostat (Leica Instruments, Nußloch, Germany). Sections were collected on Super Frost Plus glass slides (Menzel, Braunschweig, Germany) in a standard sequence, so that four sections $250 \mu \mathrm{m}$ apart were present on each slide. Immunohistochemistry was performed according to a published protocol [27]. The commercially available goat anti-choline acetyltransferase antibodies (ChAT, 1:100; Chemicon, Hofheim, Germany) were used at optimal dilutions. Water-bath antigen unmasking was performed in $0.01 \mathrm{M}$ sodium citrate solution, $\mathrm{pH} 9.0$, for $30 \mathrm{~min}$ at $80^{\circ} \mathrm{C}$ [28]. Nonspecific binding was blocked using 5\% normal serum from the species in which the secondary antibody was produced, diluted in phosphate-buffered saline, pH 7.3 (PBS), and supplemented with $0.2 \%$ Triton $\mathrm{X}-100$ and $0.02 \%$ sodium azide for $1 \mathrm{~h}$ at RT. Incubation with the primary antibody, diluted in PBS containing 0.5\% lambda-carrageenan (Sigma) and $0.02 \%$ sodium azide, was performed for three days at $4^{\circ} \mathrm{C}$. After washing in PBS (three times for $15 \mathrm{~min}$ at RT), the appropriate Cy3 conjugated secondary antibody, diluted $1: 200$ in PBS-carrageenan solution, was applied for $2 \mathrm{~h}$ at RT. Following a subsequent wash in PBS, cell nuclei were stained for $10 \mathrm{~min}$ at RT with bisbenzimide solution (Hoechst dye $33258,5 \mu \mathrm{g} / \mathrm{mL}$ in PBS; Sigma). Finally, the sections were washed again, mounted in antifading medium (Fluoromount G; Southern Biotechnology Associates via Biozol, Eching, Germany), and stored in the dark at $4^{\circ} \mathrm{C}$.

To estimate the densities of projecting immunolabeled fibers, pictures were taken on a fluorescent microscope (DM4000 Leica) with a 40x objective. Images from the RS and following subdivisions and layers of the hippocampus were taken: stratum moleculare, stratum granulosum, and stratum polymorphe of the dentate gyrus; stratum oriens, stratum pyramidale, and stratum lucidum of the CA3 region; and stratum oriens, stratum pyramidale, and stratum radiatum of the CA1 region. For each animal and layer, four pictures were taken. To estimate fiber density, the images were overlaid with a $1 \mathrm{~cm}$ stereological test grid (grid C4) using Photoshop 7.0 software (Adobe, San Jose, CA), and the number of intersections of fibers with the grid was counted.

\section{Results}

3.1. The Acetylcholinesterase Activity Is Differentially Changed in the Cortex and Hippocampus of Maternally Deprived Rats. The acetylcholinesterase activity in the synaptosomal fraction of cortex (Figure 1(a)) of MD rats was significantly decreased $(P<0.05)$ while in the hippocampus (Figure 1(b)) 


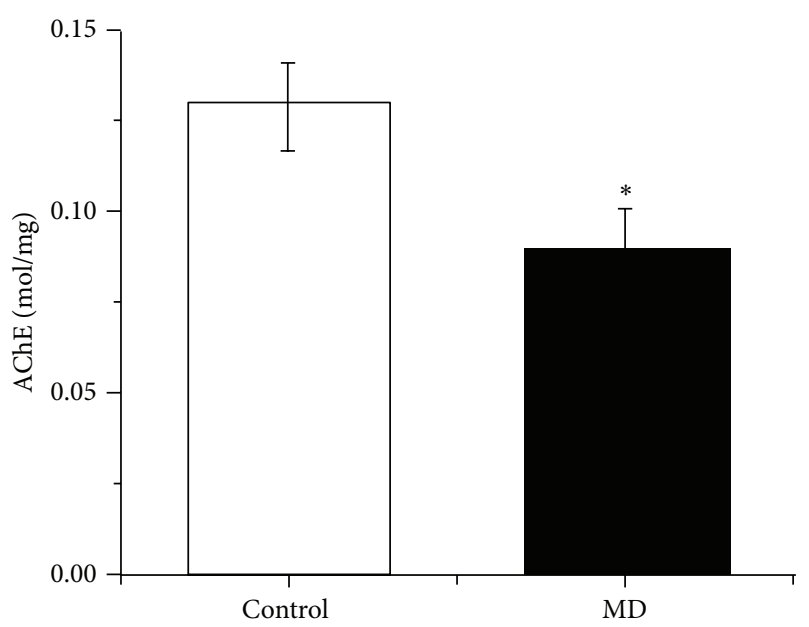

(a)

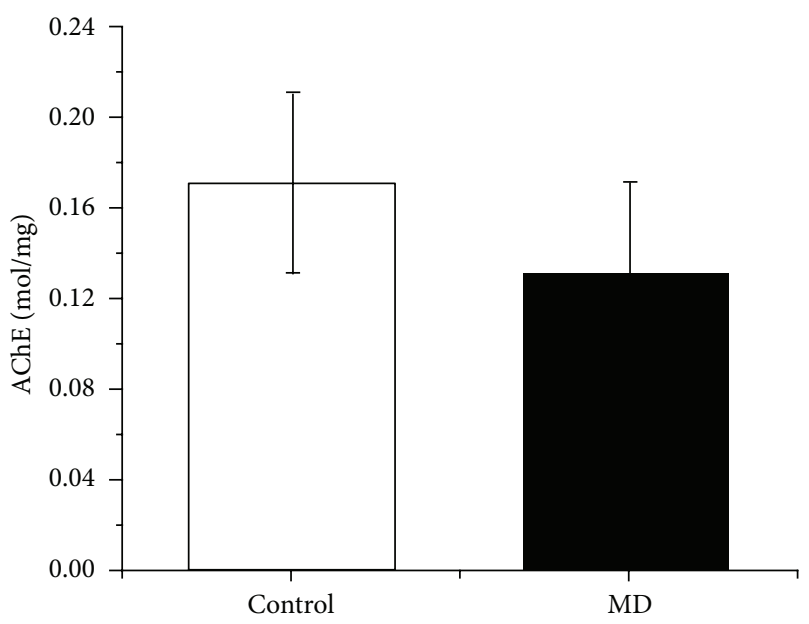

(c)

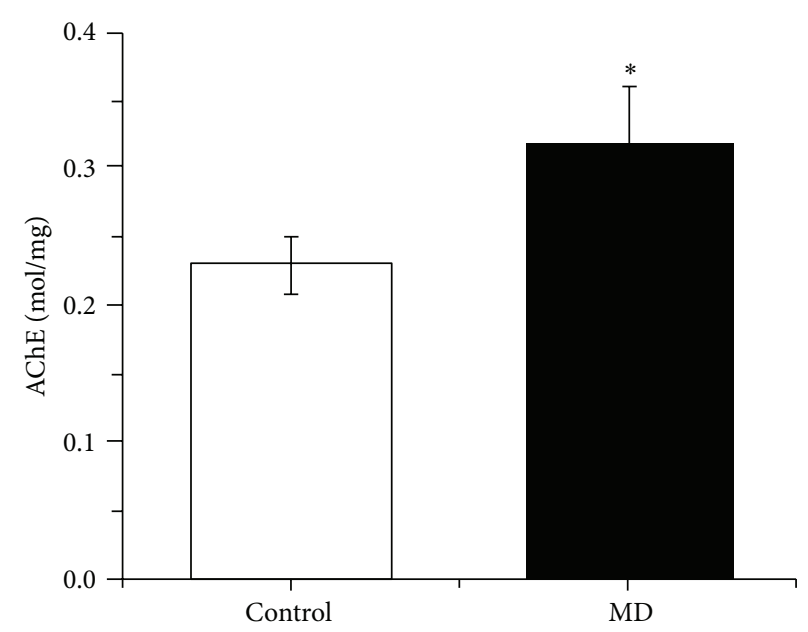

(b)

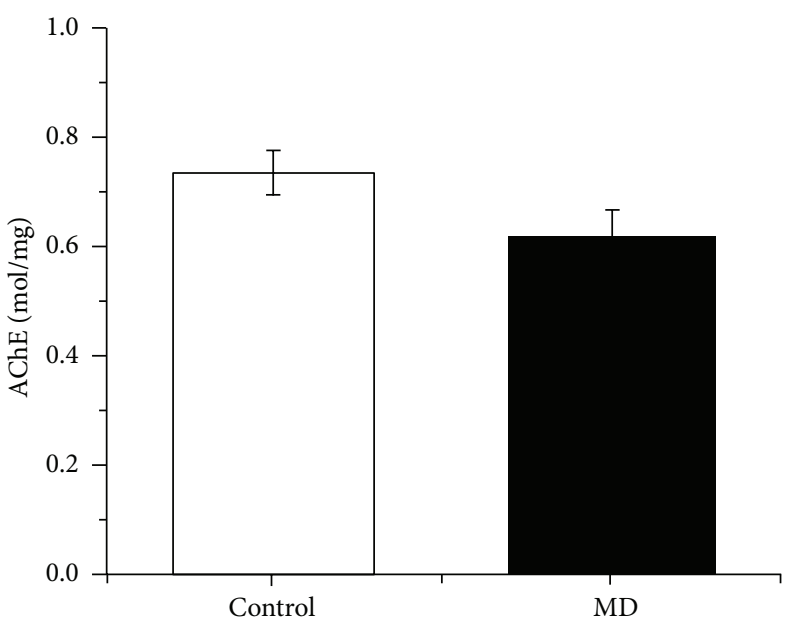

(d)

FIGURE 1: The activity of AChE in MD and control groups of animals (P 60) in synaptosomal fraction in cortex (a), hippocampus (b), thalamus (c), and caudate nuclei (d). Results are presented as mean \pm SE. ${ }^{*} P<0.05$.

it significantly increased $(P<0.05)$ comparing to the values measured in the control group. In thalamus and caudate nuclei, no change in the acetylcholinesterase activity was observed (Figures 1(c) and 1(d)).

\subsection{Immunohistochemistry Revealed Increase of the ChAT} Positive Fibers Density in the Hippocampal CA1 Sector and Decrease in RS Cortex of MD Rats. Representative immunohistochemical staining of the ChAT positive fibers in the hippocampus is presented in Figure 2(a). Measurements of ChAT positive fibers density have shown significant increase in CA1 region while no change in CA3 and DG was noticed (Figure 2(b)). The density of ChAT positive fibers in RS cortex was significantly decreased (Figure 2(c)) in MD animals.

\section{Discussion}

The results of our study have revealed region-specific changes in the AChE activity in cortex and hippocampus in the maternally separated rats. Also, the density of cholinergic fibers was decreased in the retrosplenial cortex but increased in CA1 region of hippocampus. These results are important since the hippocampus and cerebral cortex are critical areas to the processes of human's memory and cognition [29]. The cholinergic system has been proposed to contribute to the pathophysiology of schizophrenia probably as a result of an imbalance between central cholinergic and dopaminergic systems [30]. Growing body of evidence showed that patients affected with schizophrenia demonstrate cognitive deficit $[31,32]$ and cholinergic abnormalities, such as reduced acetylcholine in different brain structures [33] as well as widespread decrease in the level of muscarinic receptors in the postmortem brain [34-36]. Cholinergic involvement in schizophrenia is further supported by the fact that muscarinic antagonists can evoke a psychotic state ("antimuscarinic psychosis/syndrome"), which includes a range of cognitive and psychotic symptoms resembling schizophrenia [37]. Muscarinic cholinergic receptors (mAChRs) are G-proteincoupled receptors for the acetylcholine and consist of five 


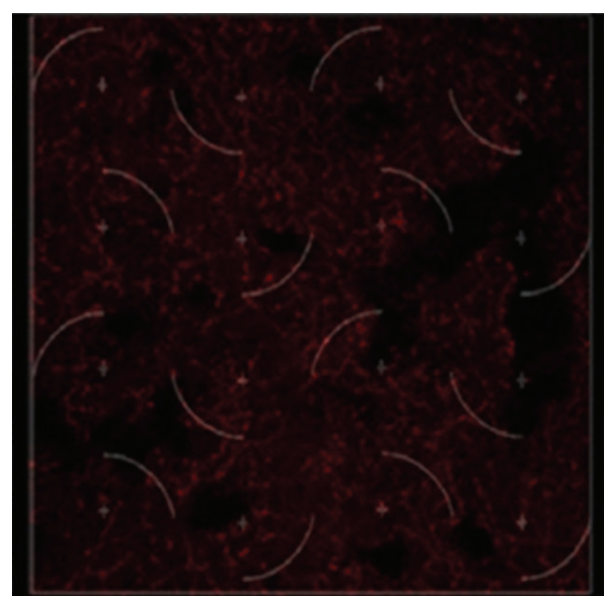

(a)

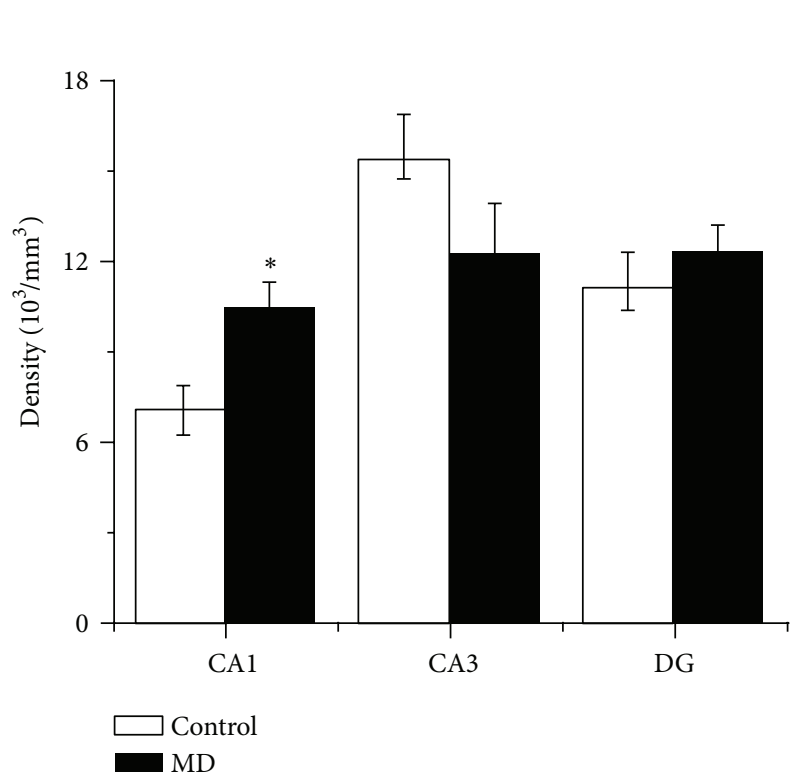

(b)

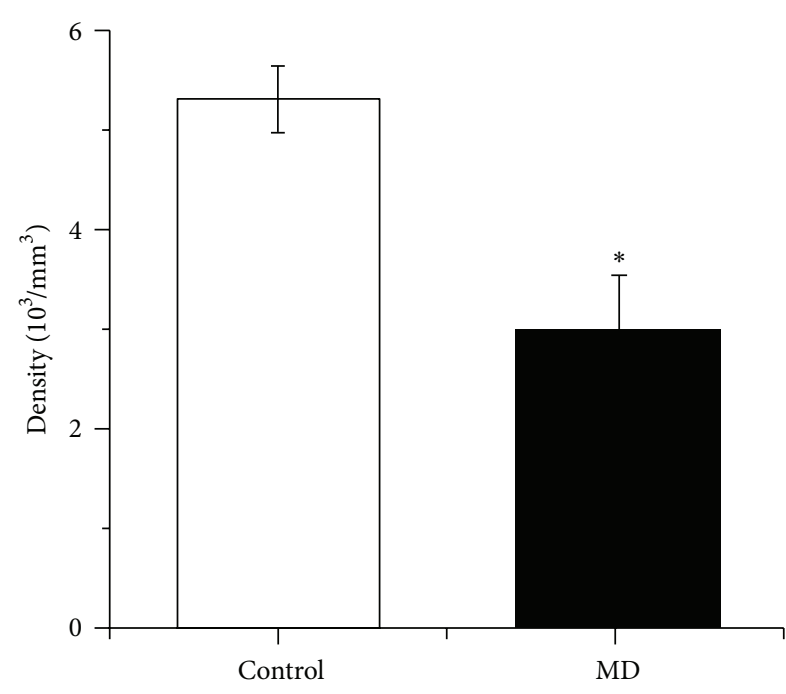

(c)

FIGURE 2: Representative immunohistochemical staining of the ChAT positive fibers in the hippocampus (a). Density of the ChAT positive fibers in the hippocampus (b) and RS cortex (c).

different subtypes, termed $\mathrm{M}_{1}-\mathrm{M}_{5}$. Numerous preclinical and clinical studies with nonselective mAChR agonists suggest that activation of $\mathrm{mAChRs}$ improves cognitive function in patients suffering from various central nervous system disorders, and these studies, along with genetic studies, indicate that $M_{1}$ is the $m A C h R$ subtype mediating the procognitive effects. Receptor protein and mRNA levels of $\mathrm{M}_{1}$ have been shown to be decreased in frontal cortex of schizophrenic patients. In addition, circulating antibodies against $M_{1}$ have been found in the serum of schizophrenics, suggesting a link between the immune system and $M_{1}$ in schizophrenics [38]. Nicotinic acetylcholine receptors (nAChRs) are ligandgated ion channels, existing as combinations from a family of similar but distinct subunits $\alpha 1-\alpha 10, \beta 1-4, \gamma, \delta$, and $\varepsilon$. The most predominant receptor in the mammalian brain is the $\alpha 4 \beta 2 \mathrm{nAChR}$, while there is also high expression of the $\alpha 7 \mathrm{nAChR}$. Both receptors are widely expressed in areas of the brain important to cognition, such as the hippocampus, thalamus, frontal, cingulate, and occipital cortices. It has been proposed that the significantly higher levels of smoking seen in patients with schizophrenia might be due to an implicit desire to activate the $\alpha 7 \mathrm{nAChR}$. Several lines of evidence suggest that the $\alpha 7$ nicotinic acetylcholine receptor (nAChR) could be an important pharmacological target for the treatment of cognitive deficits in schizophrenia. Polymorphisms in the promoter region of the $\alpha 7 \mathrm{nAChR}$ gene have been linked to sensory gating deficits in schizophrenia and some studies have found a reduced expression of $\alpha 7 \mathrm{nAChRs}$ in the frontal cortex of patients with schizophrenia [39].

On the other hand, disturbances of cholinergic system have been shown in animal models of schizophrenia. Treatment of adult rats with phencyclidine (PCP), antagonist 
of NMDA glutamate receptors, used to mimic some signs and symptoms of schizophrenia, was followed by increased efflux of acetylcholine [40] and alterations in the behaviors modulated by muscarinic receptors [41]. Furthermore, Du Bois et al. [42] have found changes in the expression of $\mathrm{M} 1 / 4$ receptors in the prefrontal cortex and hippocampus at different developmental time points following perinatal PCP treatment. Recently, Zugno et al. [43] have demonstrated that three hours of maternal deprivation daily, during first ten postnatal days (P 1 to $\mathrm{P} 10$ ), caused an increase of AChE activity in prefrontal cortex, hippocampus, and striatum, as well as behavior alterations in adult animals. The authors have also investigated the effects of three different doses of ketamine in adult rats that were perinatally exposed to maternal deprivation and found that perinatal maternal deprivation made the animals susceptible to ketamine effects. In our study, the maternal deprivation on P 9, which lasted 24 hours, has produced different effects. We have noticed decrease of AChE activity in cortex and increase in hippocampus. Furthermore, our study has demonstrated significant increase of the ChAT positive fibers density in RS cortex and selectively in the hippocampal CA1 sector. The different pattern of AChE activity changes, seen in our study and the study of Zugno et al. [43], indicates that different protocols of maternal separations are reflected on the biochemical changes in the brain of adult animals. We can suppose that the different timing of early stress could be followed by different biochemical changes and interfere with developmental process. The alterations of AChE activity are particularly important since, independently of its catalytic function, AChE exhibits multiple biological actions on neuronal cell differentiation, adhesion, and neuritogenesis [44].

On the other hand, overexpression of AChE disrupts the glutamatergic system and results in damage of synaptic structures and excitatory function [45]. All of these findings indicate that excess of $\mathrm{AChE}$ that emerges under conditions of stress [46] could contribute to the pathophysiological processes developed after procedure of maternal separation.

Our study is in agreement with the findings of cholinergic abnormalities in schizophrenia and further supports the need for investigation of therapeutic strategies involving this neurotransmitter system. Whereas drugs, which have specific dopaminergic system as targets, are effective in reducing the positive symptoms in schizophrenia, they are not sufficient against the negative symptoms and cognitive disorder [47]. The neurotransmitter acetylcholine which plays an important role in the cognitive processes $[11,48]$ and learning and memory functions $[49,50]$ can play a key role in the development of new drugs. Use of acetylcholinesterase (AChE) inhibitors represents a promising possibility for treatment of cognitive disorder in schizophrenia and other psychiatric disorders [51].

However further experiments that will include the measurement of the density of cholinergic fibers in other cortical regions especially frontal cortex, the density of neuronal cell bodies in the nucleus basalis Meynert, and the density of $\mathrm{mAChR}$ and $\mathrm{nAChR}$ are needed for better understanding of the long-lasting cholinergic system changes initiated with early temporarily maternal separation.

\section{Conclusion}

Maternal deprivation causes region-specific changes of the AChE activity and cholinergic fiber density. In the cortex, the activity of AChE and the density of ChAT positive fibers were decreased while in the hippocampus AChE activity in whole structure and the density of ChAT positive fibers in CA1 region were increased. These results are important since the hippocampus and cerebral cortex are critical areas to the processes of human's memory and cognition and further support the need for investigations of therapeutic strategies involving cholinergic system in the treatment of schizophrenia.

\section{Conflict of Interests}

The authors declare that there is no conflict of interests regarding the publication of this paper.

\section{Authors' Contribution}

All authors have read and approved the final paper.

\section{Acknowledgment}

This study was supported by Grants 175058 and III 41020 from the Ministry for Science and Environmental Protection of the Republic of Serbia.

\section{References}

[1] M. Koehl, V. Lemaire, M. Vallée et al., "Long term neurodevelopmental and behavioral effects of perinatal life events in rats," Neurotoxicity Research, vol. 3, no. 1, pp. 65-83, 2001.

[2] M.-P. Viveros, F. Díaz, B. Mateos, N. Rodríguez, and J. A. Chowen, "Maternal deprivation induces a rapid decline in circulating leptin levels and sexually dimorphic modifications in hypothalamic trophic factors and cell turnover," Hormones and Behavior, vol. 57, no. 4-5, pp. 405-414, 2010.

[3] E. F. Walker and D. Diforio, "Schizophrenia: a neural diathesisstress model," Psychological Review, vol. 104, no. 4, pp. 667-685, 1997.

[4] B. A. Ellenbroek and A. R. Cools, "The neurodevelopmental hypothesis of schizophrenia: clinical evidence and animal models," Neuroscience Research Communications, vol. 22, no. 3, pp. 127-136, 1998.

[5] E. Bramon, J. Kelly, J. van Os, and R. M. Murray, "The cascade of increasingly deviant development that culminates in the onset of schizophrenia," NeuroScience News, vol. 4, no. 1, pp. 5-19, 2001.

[6] D. J. Foster, C. K. Jones, and P. J. Conn, "Emerging approaches for treatment of schizophrenia: modulation of cholinergic signaling," Discovery Medicine, vol. 14, no. 79, pp. 413-420, 2012.

[7] D. J. King, "Drug treatment of the negative symptoms of schizophrenia," European Neuropsychopharmacology, vol. 8, no. 1, pp. 33-42, 1998.

[8] R. Tandon, J. E. Shipley, J. F. Greden, N. A. Mann, W. H. Eisner, and J.-A. Goodson, "Muscarinic cholinergic hyperactivity in schizophrenia. Relationship to positive and negative symptoms," Schizophrenia Research, vol. 4, no. 1, pp. 23-30, 1991. 
[9] R. Freedman, A. Olincy, R. W. Buchanan et al., "Initial phase 2 trial of a nicotinic agonist in schizophrenia," The American Journal of Psychiatry, vol. 165, no. 8, pp. 1040-1047, 2008.

[10] E. Scarr, "Muscarinic receptors: their roles in disorders of the central nervous system and potential as therapeutic targets," CNS Neuroscience \& Therapeutics, vol. 18, no. 5, pp. 369-379, 2012.

[11] P. E. Gold, "Acetylcholine modulation of neural systems involved in learning and memory," Neurobiology of Learning and Memory, vol. 80, no. 3, pp. 194-210, 2003.

[12] E. Perry, M. Walker, J. Grace, and R. Perry, "Acetylcholine in mind: a neurotransmitter correlate of consciousness?" Trends in Neurosciences, vol. 22, no. 6, pp. 273-280, 1999.

[13] A. Shekhar, W. Z. Potter, J. Lightfoot et al., "Selective muscarinic receptor agonist xanomeline as a novel treatment approach for schizophrenia," The American Journal of Psychiatry, vol. 165, no. 8, pp. 1033-1039, 2008.

[14] A. Fine, C. Hoyle, C. J. Maclean, T. L. LeVatte, H. F. Baker, and R. M. Ridley, "Learning impairments following injection of a selective cholinergic immunotoxin, ME20.4 IgG-saporin, into the basal nucleus of Meynert in monkeys," Neuroscience, vol. 81, no. 2, pp. 331-343, 1997.

[15] M. I. Miranda and F. Bermúdez-Rattoni, "Reversible inactivation of the nucleus basalis magnocellularis induces disruption of cortical acetylcholine release and acquisition, but not retrieval, of aversive memories," Proceedings of the National Academy of Sciences of the United States of America, vol. 96, no. 11, pp. 6478-6482, 1999.

[16] D. J. Bucci, P. C. Holland, and M. Gallagher, "Removal of cholinergic input to rat posterior parietal cortex disrupts incremental processing of conditioned stimuli," The Journal of Neuroscience, vol. 18, no. 19, pp. 8038-8046, 1998.

[17] M. Sarter and J. P. Bruno, "Cognitive functions of cortical acetylcholine: toward a unifying hypothesis," Brain Research Reviews, vol. 23, no. 1-2, pp. 28-46, 1997.

[18] E. Lucas-Meunier, P. Fossier, G. Baux, and M. Amar, "Cholinergic modulation of the cortical neuronal network," Pflugers Archiv, vol. 446, no. 1, pp. 17-29, 2003.

[19] M. B. Newman, S. J. Nazian, P. R. Sanberg, D. M. Diamond, and R. D. Shytle, "Corticosterone-attenuating and anxiolytic properties of mecamylamine in the rat," Progress in NeuroPsychopharmacology \& Biological Psychiatry, vol. 25, no. 3, pp. 609-620, 2001.

[20] J. F. López, H. Akil, and S. J. Watson, "Neural circuits mediating stress," Biological Psychiatry, vol. 46, no. 11, pp. 1461-1471, 1999.

[21] A. V. Terry Jr. and S. P. Mahadik, “Time-dependent cognitive deficits associated with first and second generation antipsychotics: cholinergic dysregulation as a potential mechanism," Journal of Pharmacology and Experimental Therapeutics, vol. 320, no. 3, pp. 961-968, 2007.

[22] M. Sarter, C. L. Nelson, and J. P. Bruno, "Cortical cholinergic transmission and cortical information processing in schizophrenia," Schizophrenia Bulletin, vol. 31, no. 1, pp. 117-138, 2005.

[23] B. A. Ellenbroek, N. M. de Bruin, P. T. van den Kroonenburg, E. L. van Luijtelaar, and A. R. Cools, "The effects of early maternal deprivation on auditory information processing in adult wistar rats," Biological Psychiatry, vol. 55, no. 7, pp. 701-707, 2004.

[24] D. V. Mićić and N. D. Petronijević, "Acetylcholinesterase activity in the Mongolian gerbil brain after acute poisoning with aluminum," Journal of Alzheimer's Disease, vol. 2, no. 1, pp. 16,2000 .
[25] D. V. Mićić, N. D. Petronijević, and S. S. Vucetić, "Superoxide dismutase activity in the mongolian gerbil brain after acute poisoning with aluminum," Journal of Alzheime's Disease, vol. 5, no. 1, pp. 49-56, 2003.

[26] V. P. Whittaker and L. A. Barker, "The subcellular fractionation of brain tissue with special reference to the preparation of synaptosomes and their component organelles," in Methods in Neurochemistry, vol. 2, pp. 1-52, Marcel Dekker, New York, NY, USA, 1972.

[27] A. Irintchev, A. Rollenhagen, E. Troncoso, J. Z. Kiss, and M. Schachner, "Structural and functional aberrations in the cerebral cortex of tenascin-C deficient mice," Cerebral Cortex, vol. 15, no. 7, pp. 950-962, 2005.

[28] Y. Jiao, Z. Sun, T. Lee et al., "A simple and sensitive antigen retrieval method for free-floating and slide-mounted tissue sections," Journal of Neuroscience Methods, vol. 93, no. 2, pp. 149-162, 1999.

[29] C. J. Morgan, A. Mofeez, B. Brandner, L. Bromley, and H. V. Curran, "Acute effects of ketamine on memory systems and psychotic symptoms in healthy volunteers," Neuropsychopharmacology, vol. 29, no. 1, pp. 208-218, 2004.

[30] R. Tandon and J. F. Greden, "Cholinergic hyperactivity and negative schizophrenic symptoms. A model of cholinergic/dopaminergic interactions in schizophrenia," Archives of General Psychiatry, vol. 46, no. 8, pp. 745-753, 1989.

[31] K. C. Zierhut, A. Schulte-Kemna, J. Kaufmann, J. Steiner, B. Bogerts, and K. Schiltz, "Distinct structural alterations independently contributing to working memory deficits and symptomatology in paranoid schizophrenia," Cortex, vol. 49, no. 4, pp. 1063-1072, 2013.

[32] K. Drusch, A. Lowe, K. Fisahn et al., "Effects of nicotine on social cognition, social competence and self-reported stress in schizophrenia patients and healthy controls," European Archives of Psychiatry and Clinical Neuroscience, vol. 263, no. 6, pp. 519527, 2013.

[33] M. Bencherif, M. K. Stachowiak, A. J. Kucinski, and P. M. Lippiello, "Alpha7 nicotinic cholinergic neuromodulation may reconcile multiple neurotransmitter hypotheses of schizophrenia," Medical Hypotheses, vol. 78, no. 5, pp. 594-600, 2012.

[34] Z. Katerina, K. Andrew, M. Filomena, and H. Xu-Feng, "Investigation of M1/M4 muscarinic receptors in the anterior cingulate cortex in schizophrenia, bipolar disorder, and major depression disorder," Neuropsychopharmacology, vol. 29, no. 3, pp. 619-625, 2004.

[35] K. A. Newell, K. Zavitsanou, S. K. Jew, and X.-F. Huang, "Alterations of muscarinic and GABA receptor binding in the posterior cingulate cortex in schizophrenia," Progress in NeuroPsychopharmacology \& Biological Psychiatry, vol. 31, no. 1, pp. 225-233, 2007.

[36] A. S. Gibbons, E. Scarr, S. Boer et al., "Widespread decreases in cortical muscarinic receptors in a subset of people with schizophrenia," The International Journal of Neuropsychopharmacology, vol. 16, no. 1, pp. 37-46, 2013.

[37] S. Barak, "Modeling cholinergic aspects of schizophrenia: focus on the antimuscarinic syndrome," Behavioural Brain Research, vol. 204, no. 2, pp. 335-351, 2009.

[38] B. J. Melancon, J. C. Tarr, J. D. Panarese, M. R. Wood, and C. W. Lindsley, "Allosteric modulation of the $\mathrm{M}_{1}$ muscarinic acetylcholine receptor: improving cognition and a potential treatment for schizophrenia and Alzheimer's disease," Drug Discovery Today, vol. 18, no. 23-24, pp. 1185-1199, 2013. 
[39] J. W. Young and M. A. Geyer, "Evaluating the role of the alpha7 nicotinic acetylcholine receptor in the pathophysiology and treatment of schizophrenia," Biochemical Pharmacology, vol. 86, no. 8, pp. 1122-1132, 2013.

[40] T. Hanania and K. M. Johnson, "Regulation of NMDAstimulated $\left[14_{\mathrm{C}}\right] \mathrm{GABA}$ and $\left[3_{\mathrm{H}}\right]$ cetylcholine release by striatal glutamate and dopamine receptors," Brain Research, vol. 844, no. 1-2, pp. 106-117, 1999.

[41] A. Mouri, Y. Noda, T. Enomoto, and T. Nabeshima, "Phencyclidine animal models of schizophrenia: approaches from abnormality of glutamatergic neurotransmission and neurodevelopment," Neurochemistry International, vol. 51, no. 2-4, pp. 173-184, 2007.

[42] T. M. du Bois, K. A. Newell, M. Han, C. Deng, and X.-F. Huang, "Perinatal PCP treatment alters the developmental expression of prefrontal and hippocampal muscarinic receptors," Progress in Neuro-Psychopharmacology \& Biological Psychiatry, vol. 33, no. 1, pp. 37-40, 2009.

[43] A. I. Zugno, I. M. de Miranda, J. Budni et al., "Efect of maternal deprivation on acetylcholinesterase activity and beahvioral changes on the ketamine-induced animal model of schizophrenia," Neuroscience, vol. 248, pp. 252-260, 2013.

[44] H. Soreq and S. Seidman, "Acetylcholinesterase-new roles for an old actor," Nature Reviews Neuroscience, vol. 2, no. 4, pp. 294302, 2001.

[45] H. Dong, Y.-Y. Xiang, N. Farchi et al., "Excessive expression of acetylcholinesterase impairs glutamatergic synaptogenesis in hippocampal neurons," The Journal of Neuroscience, vol. 24, no. 41, pp. 8950-8960, 2004.

[46] I. Nijholt, N. Farchi, M. Kye et al., "Stress-induced alternative splicing of acetylcholinesterase results in enhanced fear memory and long-term potentiation," Molecular Psychiatry, vol. 9, no. 2, pp. 174-183, 2004.

[47] C. S. Karam, J. S. Ballon, N. M. Bivens et al., "Signaling pathways in schizophrenia: emerging targets and therapeutic strategies," Trends in Pharmacological Sciences, vol. 31, no. 8, pp. 381-390, 2010.

[48] B. Moghaddam and M. E. Jackson, "Glutamatergic animal models of schizophrenia," Annals of the New York Academy of Sciences, vol. 1003, pp. 131-137, 2003.

[49] S. Benetti, A. Mechelli, M. Picchioni, M. Broome, S. Williams, and P. McGuire, "Functional integration between the posterior hippocampus and prefrontal cortex is impaired in both first episode schizophrenia and the at risk mental state," Brain, vol. 132, no. 9, pp. 2426-2436, 2009.

[50] R. W. Brown, K. S. Beale, and G. D. J. Frye, "Mecamylamine blocks enhancement of reference memory but not working memory produced by post-training injection of nicotine in rats tested on the radial arm maze," Behavioural Brain Research, vol. 134, no. 1-2, pp. 259-265, 2002.

[51] E. Giacobini, "Cholinesterase inhibitors: new roles and therapeutic alternatives," Pharmacological Research, vol. 50, no. 4, pp. 433-440, 2004. 

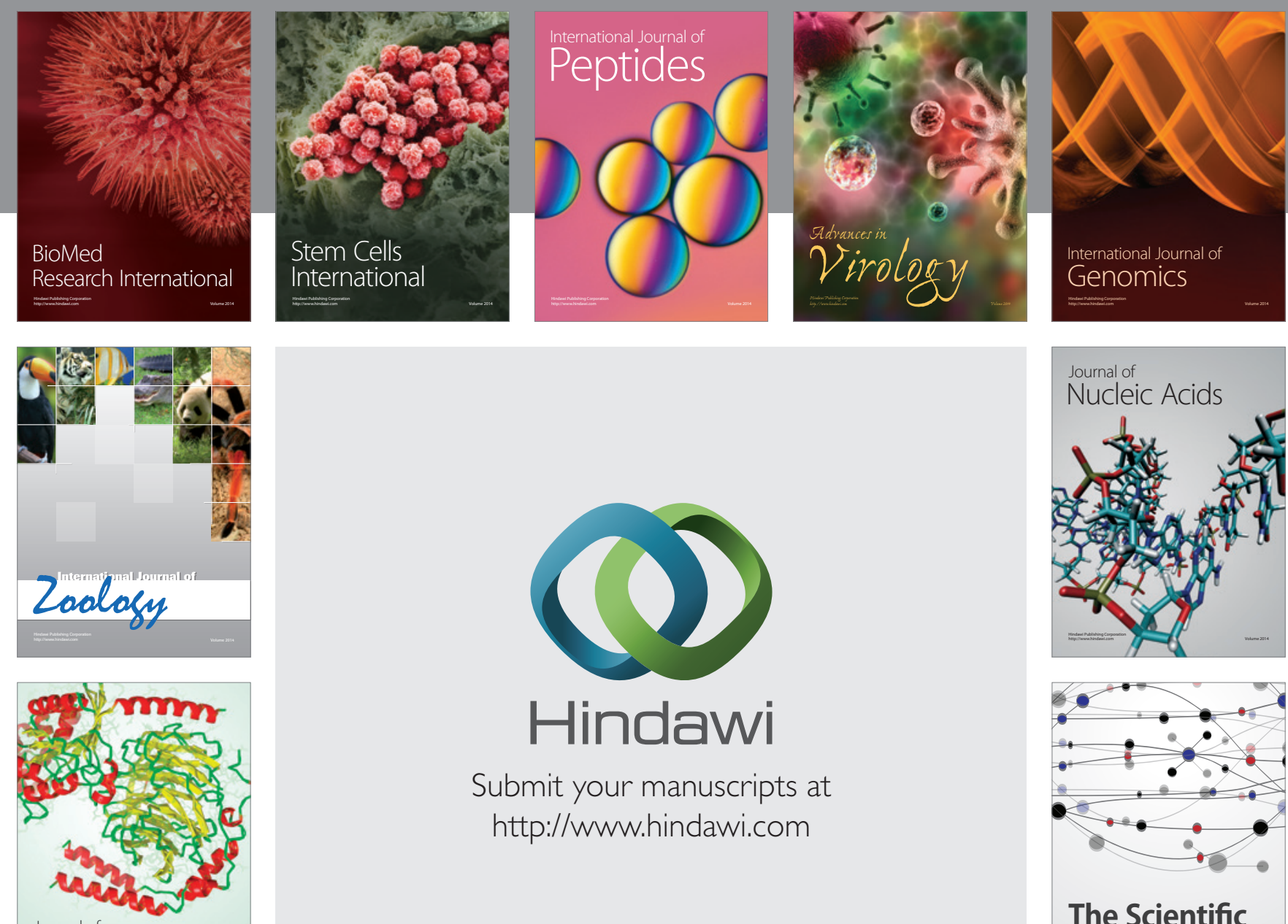

Submit your manuscripts at

http://www.hindawi.com

Journal of
Signal Transduction
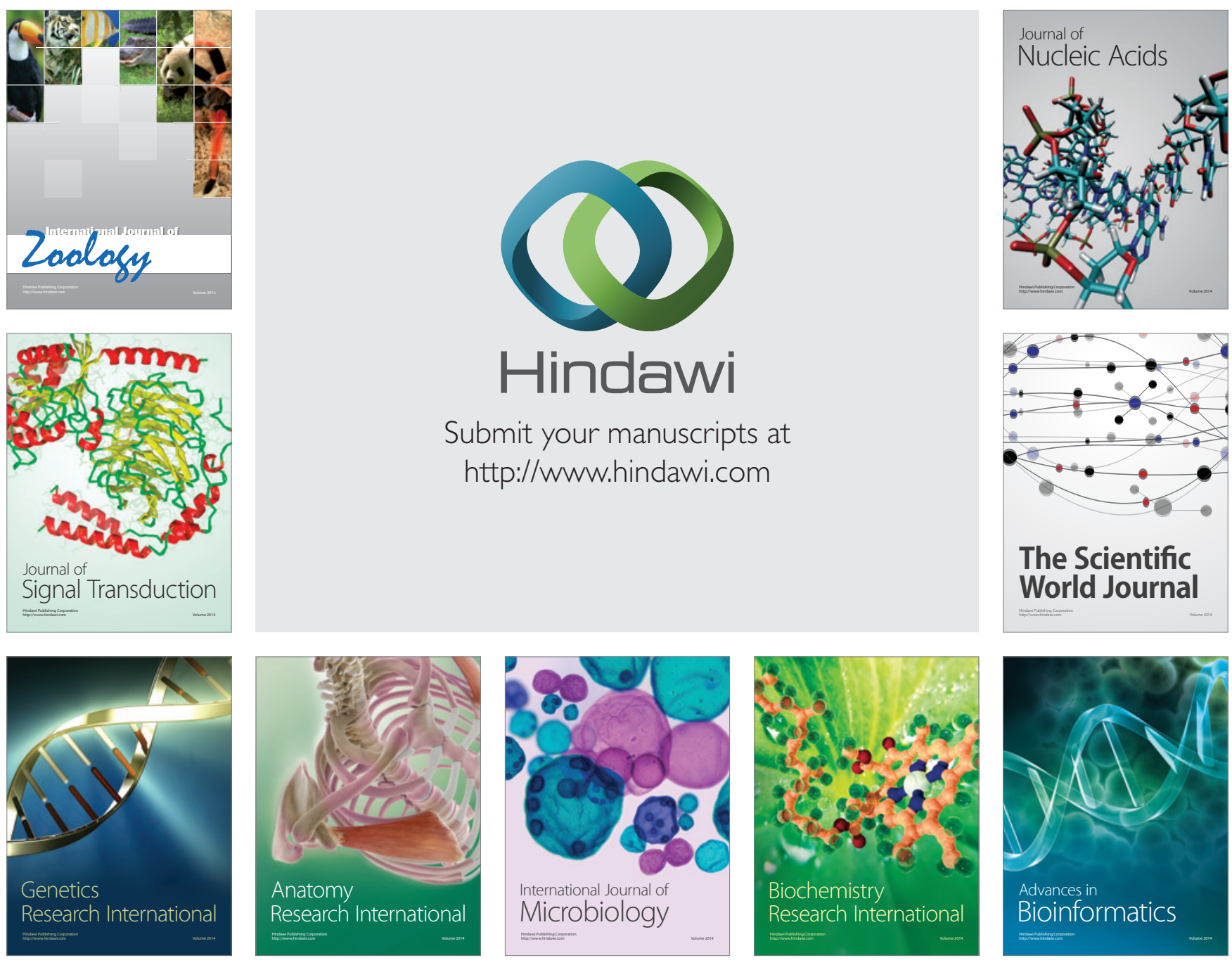

The Scientific World Journal
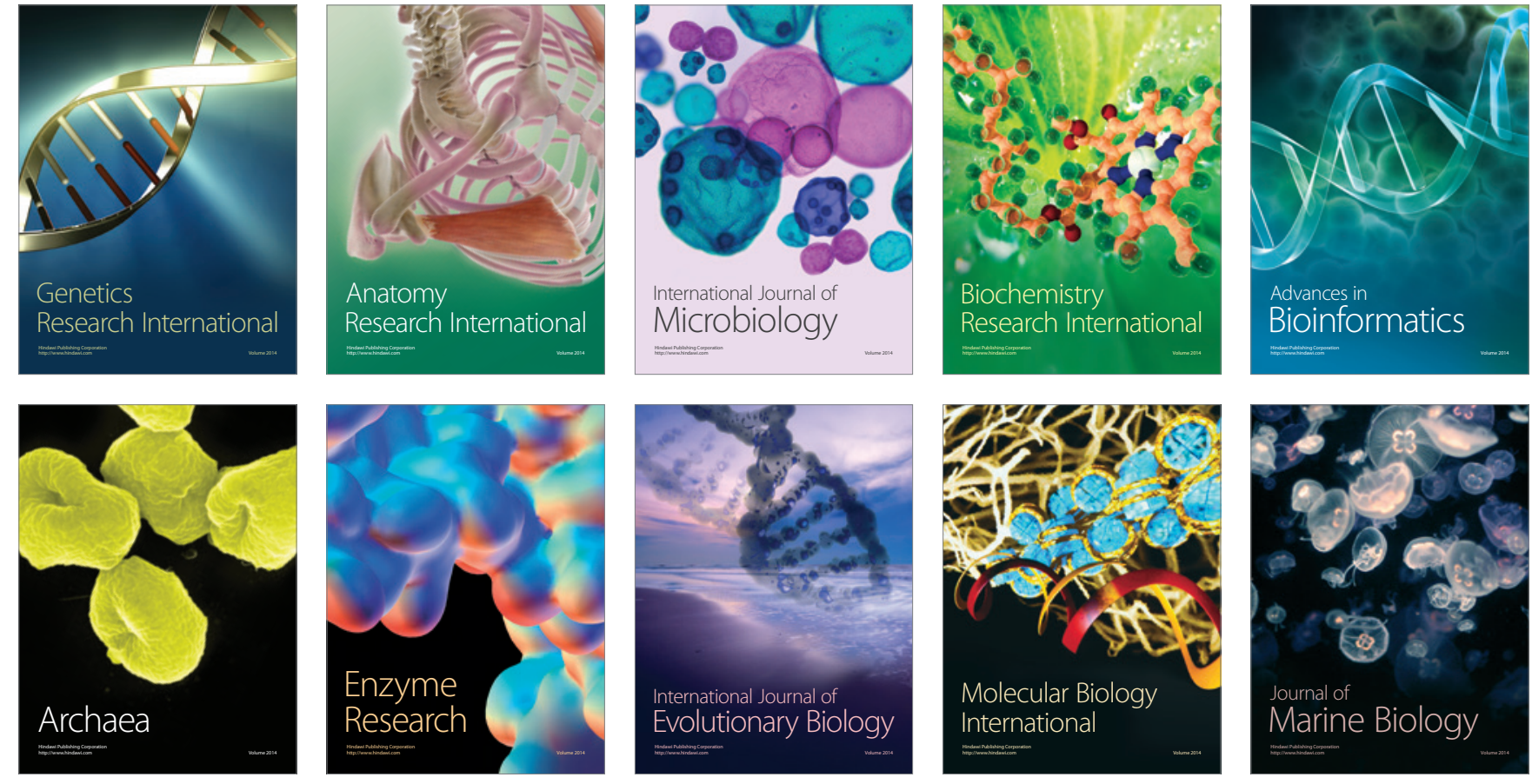